\title{
The influence of loneliness on academic, social, and emotional self-efficacy in early adolescence: A twelve month follow-up study
}

\author{
James R Andretta ${ }^{1}$ and Michael T McKay ${ }^{2 *}$ \\ ${ }^{1}$ Child Guidance Clinic, Superior Court of the District of Columbia, Washington DC, USA \\ ${ }^{2}$ Department of Psychological Sciences, University of Liverpool, Liverpool, UK
}

\begin{abstract}
The present study examined the prospective impact of loneliness in early adolescence. The study included two independent samples in the United Kingdom, Sample 1 consisted of 996 adolescents (42.67\% females, 1.7\% unreported) in Northern Ireland, while Sample 2 consisted of 829 adolescents (54.52\% females, 1.4\% unreported) in Scotland. Results showed internally consistent scores at both time points, and measurement of loneliness was invariant across SES and region groups. While scores on the UCLA Loneliness Scale - Short form retained both the same factor structure and metric across data collections, adolescents reported systematically higher scores at 12 months than baseline. Last, UCLA short scores appeared to be on both a different metric and scale between the genders. With regard to outcomes, loneliness was not shown to predict academic or emotional self-efficacy well. Loneliness was, however, inversely associated with social self-efficacy, and this effect was particularly strong in females.
\end{abstract}

\section{Introduction}

Adolescence is a period during which biological, social and psychological change can result in feelings of stress [1], and for some, loneliness [2]. Loneliness has been defined as the negative emotional response to a discrepancy between the desired and achieved quality and quantity of one's social network [3]. Accordingly, because adolescents differ in the extent to which they are able to establish intimate and satisfying peer relationships, they are also likely to differ in terms of loneliness [4]. Unfortunately, the experience of loneliness among adolescents is not uncommon [5,6] with one review reporting that approximately $80 \%$ report having felt lonely at least once [7].

The developmental course of loneliness is not entirely clear. What is known is that, with age, children become increasingly aware and concerned about being accepted by their peer group [8], and peer rejection is related to feelings of loneliness. By adolescence, concerns about one's standing within the social group emerge (Crone and Dahl 2012), and this is a strong explanatory factor in loneliness. In fact, a lack of friends, low friendship quality, peer rejection, and victimization are all predictors of loneliness in adolescence [9]. While it appears that perceived loneliness decreases towards late adolescence [4], substantive heterogeneity in the course of loneliness has been reported $[4,10,11]$.

With regard to outcomes, a growing body of research has evidenced the relationship between higher levels of loneliness throughout adolescence and unfavourable developmental sequelae across a range of domains. For instance, higher levels of loneliness have been associated with lower levels of psychological adaptation and greater incidence of clinical problems, including depression, anxiety, and suicidal ideation [4,5,12-14].

There is also reason to believe that loneliness is associated with management of social, emotional, and academic demands. That is, longitudinal studies have shown that elevated and expanding levels of loneliness are predictive of less favorable academic outcomes, including a reduced likelihood of having passed exams [15]. Those who experience problematic loneliness trajectories have also reported low levels of agreeableness, relatively deficient emotional stability, and less than adequate self-esteem $[4,13]$ as well as impaired emotional regulation [16]. Conversely, those with stable and minimal experiences of loneliness present with the most positive peer functioning [10], as well as both the most optimal personality traits (i.e., most extraverted, agreeable, and emotionally stable; [4]) and psychosocial profiles (i.e., highest self-esteem and lowest levels of perceived stress, depressive symptoms, and anxiety; [4]).

Turning to demographic covariates, previous studies on the relationship between loneliness and both socioeconomic status (SES), and gender, have yielded equivocal results $[4,15,17,13]$. Some longitudinal studies, involving the periods between childhood and the onset of early adolescence [10], and from mid-adolescence to early adulthood [4], have revealed no differences in loneliness trajectories by gender. On the other hand, a latent class study of Latino adolescents in the first two years of high school reported gender differences in the trajectory of loneliness [15]. That is, males were most likely to present with relatively low levels of loneliness that increased over time. SES

*Correspondence to: Michael McKay, Department of Psychological Sciences, University of Liverpool, Bedfprd Street South, Liverpool, BT60 7ZA, UK, E-mail: Michael.Mckay@liverpool.ac.uk

Key words: loneliness, self-efficacy, confirmatory factor analysis (CFA), crossnational

Received: August 21, 2018; Accepted: September 14, 2018; Published: September 18, 2018 
has previously been related to loneliness levels, with higher levels of loneliness observed in lower SES groups. Elsewhere, Vanhalst, et al. [4] reported no meaningful differences in loneliness trajectory classes across SES groups.

In terms of cross-cultural research on loneliness, the literature is a lot more far sparse [18], and no consensus exists regarding the prevalence of loneliness as a function of individualistic versus collectivistic cultures [19]. Indeed, Van Staden and Coetzee [20] recommended that crosscultural studies should also be conducted within countries to examine loneliness in subcultures and minority groups. Given the limited knowledge and agreement about demographic variability in loneliness, the present study sought to address potential differences in both the measurement and effects of loneliness between the genders, SES groups (i.e., free school meal provided v. not eligible), and geographic regions (i.e., Scotland v. Northern Ireland).

There is also reason to believe that loneliness is associated with selfefficacy: "people's judgments of their capabilities to organize and execute courses of action required to attain designated types of performance" [21], or again, "self-efficacy beliefs determine how people feel, think, motivate themselves and behave" [22]. Accordingly, these self-efficacy beliefs help determine individuals' choices, efforts, persistence, and perseverance in tasks. Efficacy beliefs are best understood as domainspecific (e.g. Muris 2001) such that self-efficaciousness in one domain (e.g. academics) does not always translate to all domains of life (e.g., emotions); therefore, feelings of competence tied to task demands of a given situation have greater predictive utility than a global selfevaluation [23].

\section{The present study}

The present study aimed to investigate a number of questions. Firstly, we aimed to examine the psychometric properties of the UCLA Loneliness Scale-Revised scores, and associated descriptive statistics, in a large sample of adolescents living in the UK across 12 months. Secondly, we sought to examine how loneliness, and the development of loneliness across that period, predicted scores on three domains of self-efficacy. We hypothesized that loneliness would have a negative association with social, emotional, and academic self-efficacy.

\section{Methods}

\section{Participants and procedure}

Data were collected in two independent samples of school children in the United Kingdom. Schools were randomly chosen to be part of a large longitudinal adolescent development study. Data were collected at baseline (participants were in school Grade 9 [aged 13-14 years]), and at +12 months. Sample 1 consisted of 996 adolescents ( $42.67 \%$ females, 1.7\% unreported; $99.0 \%$ Caucasian) attending secondary schools in Northern Ireland. Sample 2 consisted of 829 adolescents $(54.52 \%$ females, $1.4 \%$ unreported; $80.5 \%$ Caucasian, $14.2 \%$ British Pakistani) attending secondary schools in Scotland. Both samples completed the same questionnaires alongside several other questionnaires as part of a large scale representative longitudinal study. A form of parental opt-out consent was approved for the study by the Ethics Board of the second author, and participants also gave informed consent at each data collection point.

\section{Measures}

Loneliness was measured using the revised three-item UCLA Loneliness Scale [24], "How often do you feel that you lack companionship?", "How often do you feel left out?", and "How often do you feel isolated from others?" The full UCLA Scale consists of 20 items; however, a previous study has shown that a short form of the scale has adequate validity for inclusion in large-scale studies [24]. Furthermore, Hughes, et al. showed a strong correlation between short and fulllength scale scores $(r=.82)$. The items were rated hardly ever $(0)$, some of the time (1) or often (2). We summed the items to produce a total loneliness score. See (Table 1) for reliability coefficients.

The Self-Efficacy Questionnaire for Children (SEQ-C) contains 21 items assessing three domains of self-efficacy: (a) academic selfefficacy (e.g., "How well do you succeed in passing all subjects?"), (b) emotional self-efficacy (e.g., "How well can you control your feelings?"), and (c) social self-efficacy (e.g., "How well do you succeed in staying friends with other children?"). Each subscale consists of seven items, and respondents rate their competence in each self-efficacy domain on a 5 -point Likert scale ( $1=$ not at all; $5=$ very well). SEQ-C subscale scores have been found to be structurally valid and internally consistent $(\alpha>80)$.

Information was gathered on gender, and free school meals entitlement (FSM), an imperfect proxy for low-income families, and thus SES [25]. The proportions for FSM for Northern Ireland and Scotland were (respectively): Yes=16.9\%/18.9\%; No=76.6\%/65.9\%; Unsure $=6.5 \% / 15.3 \%$.

We examined the model fit for the UCLA Loneliness Scale, and performed conducted Structural Equation Modelling (SEM) based on a covariance matrix and used maximum likelihood robust (MLR) estimation in MPLUS 7.1 [26], which is robust to missing values and non-normality. CFA was employed to examine how well the UCLA short form theory fit data in adolescents, with the following guidelines applied: (a) comparative fit indices of CFI $\geq .95$ and TLI $\geq .90$, (b) absolute misfit indices of RMSEA $\leq .06$ and SRMR $\leq .08$ [27].

SEM models were developed to examine path coefficients between loneliness scores at baseline and outcomes +12 months. All SEM models were developed to control for clustering at the school level, and crosslagged panel designs were employed to control for variance attributed to stability (i.e., autoreggression among variables; [28]. Because some individuals participated in an alcohol intervention during the course of data collection, this variable was controlled for in all analyses as well.

Last, multi-group SEM models were employed using maximum likelihood parameter estimates with standard errors and a mean- and variance-adjusted $\chi^{2}$ test statistics that are robust to non-normality (MLMV) estimation to test invariance of structural path coefficients across groups. The goal here was to test whether or not the direct

Table 1. Correlations and descriptive statistics

\begin{tabular}{|c|c|c|c|c|c|}
\hline Variable & 1 & 2 & 3 & 4 & 5 \\
\hline 1. Loneliness $\mathrm{T} 1$ & -- & $0.35^{* *}$ & $-0.15^{* *}$ & $-0.25^{* *}$ & $-0.24 * *$ \\
\hline 2. Loneliness $\mathrm{T} 2$ & $0.51 * *$ & -- & $-0.23 * * a$ & $-.27 * * a$ & $-0.33 * * a$ \\
\hline 3. Academic Self-Efficacy & -0.07 & $-0.10^{* a}$ & -- & $.36 * *$ & $0.36^{* *}$ \\
\hline 4. Social Self-Efficacy & $-0.27 * *$ & $-0.23 * * a$ & $.30 * *$ & -- & $0.55^{* *}$ \\
\hline 5. Emotional Self-Efficacy & $-0.33 * *$ & $-0.31 * * a$ & $0.30 * *$ & $0.52 * *$ & -- \\
\hline$\alpha(95 \% \mathrm{XI} \varepsilon)$ & $0.79(0.78)$ & $0.78(0.76)$ & $0.86(0.85)$ & $0.77(0.75)$ & $0.87(0.86)$ \\
\hline$M(\mathrm{SD})$ & $0.50(0.51)$ & $0.56(0.55)$ & $3.41(0.78)$ & $3.64(0.65)$ & $3.12(0.84)$ \\
\hline Irtosis & 3.24 & 2.74 & 2.96 & 3.71 & 2.72 \\
\hline Skewness & 0.92 & 0.74 & -0.4 & -0.48 & -0.13 \\
\hline \multicolumn{6}{|c|}{$\begin{array}{l}\text { Note. } * \leq .05 * * \leq .001 \text {. Correlations for the Northern Ireland sample below, and Scottish } \\
\text { sample above, the diagonal. Descriptive statistics included the entire sample. Power } \\
\text { analyses using the } p w r \text { package in } R \text { statistics showed that a } r \text { coefficient of } .10 \text { was the } \\
\text { cutoff for a minimally interpretable correlation. Power was set to the recommended level } \\
\text { of } .80 \text { (Ellis, 2010), and significance level was determined using Bonferroni's adjustment } \\
\text { (i.e., } p=.001) \text {. a=partial correlation controlling for loneliness T1 scores. }\end{array}$} \\
\hline
\end{tabular}


and indirect effects of loneliness on covariates were invariant between the genders. In application, a scaled difference in $\chi^{2}$ test was used to determine whether or not gender and or SES moderated the effect of loneliness scores at baseline on outcomes +12 months. To aid the interpretation of results we employed Ferguson's [29] recommendations for the interpretation of effect sizes. Accordingly a correlation coefficient of $>.2$ was interpreted as practically significant, $>.50$ as moderate, and $>.80$ as a large effect size. Additionally a Cohen's $d$ of $>.41$ was interpreted as practically significant.

\section{Results}

Descriptive statistics are shown in (Table 1), where virtually no attrition was observed for loneliness scores between baseline and +12 months. Attrition by outcome is reported in the table notes. Results showed adequate internal consistency of scores across constructs, and none of the measurement scores were skewed or kurtotic. By region and SES (Table 2), configural, weak, strong, and strict measurement invariance of loneliness scores were observed. Therefore, tests of structural invariance between the SES and region groups can be made without caution. Results by gender showed a lack of measurement invariance. Configural invariance was observed by gender, meaning that the same number of short UCLA factors exist in males and females. However, weak, strong, and strict invariance of scores were not observed. These data show that UCLA short form measurement in males is not on the same scale as females (weak invariance), which means the scores are not on the same metric and thereby weak invariance was not met. Consequently, gender comparisons of underlying factor scores between the genders should be made with caveat that there are substantive differences in the measurement of loneliness between males and females. A test of strong invariance further showed that females $(M$ for three items $=.525, .697$, and .519$)$ responded systematically higher items when compared to males ( $M$ for three items $=.387, .506$, and .368 ), and therefore UCLA short form scores do not appear to have the same scalar between the genders. It is noted that the lack of scalar invariance was in keeping with theory, where girls have been shown to report more loneliness than boys during adolescence.

Invariance of UCLA short scores was also examined between data collections (Table 3). Results were not entirely in keeping with theory. It was observed that loneliness scores maintained the same factor structure (configural) across data collections, and that scores remained on the same metric (weak or metric invariance). However, UCLA short scores did not retain the same scalar, and that was because adolescents reported systematically higher scores at wave $2(M$ for three items $=.543$, .657 , and .477) than wave 1 ( $M$ for three items $=.453, .598$, and .441 ).

An Analysis of Variance (ANOVA) was developed to further examine the effect of gender and SES on loneliness at baseline: $F$ $(2,1565)=20.12, p \leq .001$, Adjusted $R^{2}=.02$. Females $(b=.16,95 \%$ confidence interval $[\mathrm{CI}]=.11-.21)$ and students who receive free meals ( $b=.05,95 \%$ confidence interval $[\mathrm{CI}]=-.01-.11)$ both reported more loneliness than their peers, with small effect sizes.

Correlations between baseline loneliness scores and the other continuous variables were computed, whereas partial correlations were used to examine correlations between loneliness +12 months and the other outcomes controlling for baseline scores (Table 1). There were no coefficients in the moderate range [29] between loneliness at either baseline or +12 months, and any other variables. A similar pattern of results emerged in both Northern Ireland and Scotland, where there

Table 2. Measurement invariance by region, gender, and SES $(\mathrm{N}=1829)$

\begin{tabular}{|c|c|c|c|c|c|c|c|c|c|}
\hline Model & $\chi^{2}$ & & $d f$ & CFI & $\Delta$ CFI & TLI & $\Delta$ TLI & SRMR & $\begin{array}{c}\text { RMSEA (90\% } \\
\text { CI) }\end{array}$ \\
\hline \multicolumn{10}{|l|}{ Region (1) } \\
\hline Configural $^{\mathrm{a}}$ & 0 & $*$ & 0 & 1 & -- & 1 & -- & 0 & $.000(.000, .000)$ \\
\hline Weak & 0.816 & & 2 & 1 & 0 & 1.003 & -0.003 & 0.007 & $.000(.000, .000$ \\
\hline Strong & 8.344 & & 5 & 0.997 & 0.003 & 0.996 & 0.004 & 0.032 & $.027(.000, .058$ \\
\hline Strict & 16.971 & $*$ & 8 & 0.992 & 0.008 & 0.994 & 0.006 & 0.037 & $.035(.010, .058$ \\
\hline \multicolumn{10}{|l|}{ Gender (2) } \\
\hline Configural $^{\mathrm{a}}$ & 0 & $*$ & 0 & 1 & -- & 1 & -- & 0 & $.000(.000, .000$ \\
\hline Weak & 4.795 & & 2 & 0.997 & 0.003 & 0.992 & 0.008 & 0.017 & $.039(.000, .086$ \\
\hline Strong & 50.931 & $*$ & 5 & 0.958 & 0.042 & 0.95 & 0.05 & 0.081 & $.100(.076, .126$ \\
\hline Strict & 66.617 & $*$ & 8 & 0.946 & 0.054 & 0.96 & 0.04 & 0.08 & $.090(.070, .110$ \\
\hline \multicolumn{10}{|l|}{ SES (3) } \\
\hline Configural $^{\mathrm{a}}$ & 0 & $*$ & 0 & 1 & -- & 1 & -- & 0 & $.000(.000, .000)$ \\
\hline Weak & 1.193 & & 2 & 1 & 0 & 1.002 & -0.002 & 0.008 & $.000(.000, .061)$ \\
\hline Strong & 5.542 & & 5 & 0.999 & 0.001 & 0.999 & 0.001 & 0.021 & $.012(.000, .052$ \\
\hline Strict & 10.932 & & 8 & 0.997 & 0.003 & 0.998 & 0.002 & 0.025 & $.022(.000, .050$ \\
\hline
\end{tabular}

Notes. * p-value $\leq .05$., (1) missing, $n=3$, (2) missing=4, (3) missing=259; Models 1-3 included loneliness at baseline scores. a=configural model with 3-items is "just-identified," which explains the 0.00 values.

Table 3. Measurement invariance by time $(\mathrm{N}=1826)$

\begin{tabular}{|c|c|c|c|c|c|c|c|c|c|}
\hline Model & $\chi^{2}$ & & $d f$ & CFI & $\triangle \mathrm{CFI}$ & TLI & $\Delta$ TLI & SRMR & $\begin{array}{l}\text { RMSEA }(90 \% \\
\text { CI) }\end{array}$ \\
\hline Time $1^{\mathrm{a}}$ & 0 & $*$ & 0 & 1 & -- & 1 & -- & 0 & $.000(.000, .000)$ \\
\hline Time $2^{\mathrm{a}}$ & 0 & $*$ & 0 & 1 & -- & 1 & -- & 0 & $.000(.000, .000)$ \\
\hline \multicolumn{10}{|c|}{ Time $1 \& 2(1)$} \\
\hline Configural $^{\mathrm{a}}$ & 0 & $*$ & 0 & 1 & -- & 1 & -- & 0 & $.000(.000, .000)$ \\
\hline Weak & 3.36 & & 2 & 0.999 & 0.001 & 0.998 & 0.002 & 0.01 & $.019(.000, .054)$ \\
\hline Strong & 21.678 & $*$ & 5 & 0.992 & 0.008 & 0.99 & 0.01 & 0.031 & $.043(.025, .062)$ \\
\hline Strict & 46.1 & $*$ & 8 & 0.981 & 0.019 & 0.986 & 0.014 & 0.034 & $.051(.037, .066)$ \\
\hline
\end{tabular}


were practically significant negative correlations between loneliness at baseline and +12 months and both social and emotional self-efficacy. In the Northern Irish sample, there was also a practically significant negative correlation between loneliness at +12 months and academic self-efficacy.

A cross-lagged panel design, structural equation model (SEM) was developed to examine the causal path between loneliness at baseline and academic, social, and emotional self-efficacy at a 12-month followup with gender, SES, and geographic region added as covariates. Results showed the model was an adequate fit for the data, $\chi^{2}(64)=300.28, p \leq$ $.001, \mathrm{CFI}=.94, \mathrm{RMSEA}=.06$ (95\% confidence interval $[\mathrm{CI}]=.05-.06)$. Results also showed that loneliness was stable across the 12 months of the study $(\gamma=.58, p<.001)$.

Confining interpretation to standardized coefficients, loneliness was shown to not have an effect on either academic self-efficacy $(\gamma=-.03$, $p=.26$ ) or social self-efficacy $(\gamma=-.08, p=.02)$, both of which were well explained by the model $\left(R^{2}=.45\right.$ and $R^{2}=.32$, respectively). In contrast, loneliness at baseline had a small and negative effect on emotional selfefficacy 12 months on $(\gamma=-.12, p=.001)$, and the model explained a meaningful amount of social efficacy scores $\left(R^{2}=.40\right)$. As reported via ANOVA, females $(\gamma=-.02, p=.06)$ and youth receiving free lunch $(\gamma=.09, p=.002)$ reported higher levels of loneliness at baseline, albeit differences were modest.

Because the effect for SES was significant, a second model was developed to examine a potential interaction between SES status and loneliness at baseline in the prediction of outcomes at +12 months. The interaction parameters were not statistically or practically significant with regard to academic self-efficacy. There were, however, negative effects on both social $(b=-.13, p=.005)$ and emotional $(b=-.23, p=.001)$ self-efficacy. A third model including interaction terms by gender showed a practically significant effect for gender with loneliness at baseline on emotional self-efficacy at +12 months $(b=-.21, p=.05)$. Specifically, being female and experiencing loneliness had a negative effect on emotional self-efficacy. Last, multiple sample analysis showed that neither gender nor SES group membership moderated the effect of loneliness at baseline on the aforementioned outcomes (Table 4). That is, the structural paths were invariant between the SES and gender groups.

Next, the first and primary SEM model was employed including just the youth from Northern Ireland, and again the model was an adequate fit for the data, $\chi^{2}(115)=3076.70, p \leq .001, \mathrm{CFI}=.93$, RMSEA $=.07(95 \%$ confidence interval $[\mathrm{CI}]=.06-.08)$. Among Northern Ireland youth, loneliness at baseline did not have an effect on academic self-efficacy $\left(\gamma=-.02, p=.58, R^{2}=.32\right)$ or social self-efficacy $\left(\gamma=-.05, p=.20, R^{2}=.30\right)$. It is noted that loneliness at baseline was an inverse indicator of emotional self-efficacy at +12 months $(\gamma=-.17, p \leq .001)$, and the model explained a substantial amount of social efficacy scores $\left(R^{2}=.39\right)$. The model fit for data collected from Scottish adolescents was adequate as well: $\chi^{2}(59)=163.09, \mathrm{p} \leq .001, \mathrm{CFI}=.94$, RMSEA $=.06$ (95\% confidence interval $[\mathrm{CI}]=.05-.07)$. Among Scottish adolescents, loneliness at baseline was not associated with academic self-efficacy $(\gamma=-.06, p=.04$, $\left.R^{2}=.40\right)$, emotional self-efficacy $\left(\gamma=-.06, p=.44, R^{2}=.41\right)$, or social selfefficacy at the 12 month follow up ( $\gamma=-.03, p \leq .12, R^{2}=.36$ ).

Results of a series of $t$-tests Table 5 showed that females reported substantially higher levels of loneliness than males at both baseline and +12 months, and that convergence in loneliness was minimal. Examination of means by gender also showed that both males and females reported higher levels of loneliness at 12 months than baseline. In contrast, males reported higher levels of both social and emotional self-efficacy than females. Academic self-efficacy was similar between
Table 4. Gender differences in loneliness and continuous outcomes

\begin{tabular}{|l|l|l|l|l|}
\hline & Males & Females & & \\
\cline { 2 - 5 } & $M(S D)$ & $M(S D)$ & $t(d f)$ & Cohen's $d(95 \% \mathrm{CI})$ \\
\hline Loneliness T1 & $0.42(.48)$ & $0.58(.53)$ & $-6.73(1823)^{* *}$ & $-0.32(-.41,-.22)$ \\
\hline Loneliness T2 & $0.48(.53)$ & $0.65(.55)$ & $-6.69(1823)^{* *}$ & $-0.31(-.41,-.22)$ \\
\hline $\begin{array}{l}\text { Academic Self- } \\
\text { Efficacy }\end{array}$ & $3.40(.79)$ & $3.42(.76)$ & $-0.83(1689)$ & $-0.05(-.14, .04)$ \\
\hline Social Self-Efficacy & $3.74(.63)$ & $3.54(.65)$ & $6.44(1681)^{* *}$ & $0.29(.19, .38)$ \\
\hline $\begin{array}{l}\text { Emotional Self- } \\
\text { Efficacy }\end{array}$ & $3.43(.76)$ & $2.78(.78)$ & $17.15(1655)^{* *}$ & $0.84(.74, .94)$ \\
\hline
\end{tabular}

** $p \leq .001$. Power analyses were developed using the $p w r$ package in $R$ for $t$-tests. Power was set to the recommended level of .80 (Ellis, 2010), and significance level was determined using Bonferroni's adjustment (i.e., $p=.008$ ). The cutoffs for minimally interpretable effect sizes were as follows with substantial effect sizes in italics above: (a) loneliness $\mathrm{T} 1=.16$, (b) loneliness $\mathrm{T} 2=.16$, (c) academic self-efficacy $=.17$, (d) social selfefficacy $=.17$, (e) emotional self-efficacy $=.17$.

Table 5. Gender differences in loneliness and continuous outcomes

\begin{tabular}{|c|c|c|c|c|}
\hline & \multirow{2}{*}{\begin{tabular}{|l|} 
Males \\
$M(S D)$ \\
\end{tabular}} & \multicolumn{3}{|l|}{ Females } \\
\hline & & $M(S D)$ & $t(d f)$ & Cohen's d $(95 \% \mathrm{CI})$ \\
\hline Loneliness T1 & $0.42(.48)$ & $0.58(.53)$ & $-6.73(1823)^{* *}$ & $-0.32(-.41,-.22)$ \\
\hline Loneliness T2 & $0.48(.53)$ & $0.65(.55)$ & $-6.69(1823)^{* *}$ & $-0.31(-.41,-.22)$ \\
\hline Sensation Seeking & $3.71(.82)$ & $3.53(.84)$ & $4.75(1816)^{* *}$ & $0.22(.12, .31)$ \\
\hline $\begin{array}{l}\text { Academic Self- } \\
\text { Efficacy }\end{array}$ & $3.40(.79)$ & $3.42(.76)$ & $-0.83(1689)$ & $-0.05(-.14, .04)$ \\
\hline Social Self-Efficacy & $3.74(.63)$ & $3.54(.65)$ & $6.44(1681)^{* *}$ & $0.29(.19, .38)$ \\
\hline $\begin{array}{l}\text { Emotional Self- } \\
\text { Efficacy }\end{array}$ & $3.43(.76)$ & $2.78(.78)$ & $17.15(1655)^{* *}$ & $0.84(.74, .94)$ \\
\hline \multicolumn{5}{|c|}{$\begin{array}{l}* * p \leq .001 \text {. Power analyses were developed using the } p w r \text { package in } R \text { for } t \text {-tests. } \\
\text { Power was set to the recommended level of } .80 \text { (Ellis, 2010), and significance level was } \\
\text { determined using Bonferroni's adjustment (i.e., } p=.008 \text { ). The cutoffs for minimally } \\
\text { interpretable effect sizes were as follows with substantial effect sizes in italics above: (a) } \\
\text { loneliness } T 1=.16 \text {, (b) loneliness } \mathrm{T} 2=.16 \text {, (c) sensation seeking=.16, (d) academic self- } \\
\text { efficacy }=.17 \text {, (e) social self-efficacy }=.17 \text {, (f) emotional self-efficacy }=.17 \text {. }\end{array}$} \\
\hline
\end{tabular}

the genders. However, the only difference that could be considered practically significant [29], is that for emotional self-efficacy.

\section{Discussion}

Results of the present study speak to the viability of the three-item UCLA loneliness scale in adolescent populations. First, the scale yielded internally consistent scores at baseline and at +12 months. Analyses also showed that scores were invariant across SES (i.e., subsidized lunch or not) and geographic location.

While UCLA short scores retained both the same factor structure and metric across data collections, adolescents reported systematically higher scores at 12 months than baseline. We hypothesized that the adolescents, would report systematically lower scores by +12 month follow-up. Therefore, the adolescents involved in the present study (mean age $=13.6$ at baseline and 14.5 at +12 months) were still in the midst of loneliness onset, rather than the abatement phase that has been observed later in adolescence. These data speak to the fact that early adolescence is likely marked by increases in loneliness, and that abatement likely occurs later in this developmental period. Last, UCLA short scores appeared to be on both a different metric and scale between the genders. Therefore, some caution should be taken when examining UCLA short factor scores between the genders.

Although previous studies have shown that loneliness has a negative effect on academic achievement [15] and emotional problems [5], loneliness in the present study was not a good predictor academic self-efficacy. The collective data suggest that loneliness is linked with academic outcomes but not necessarily an individual's appraisal of his or her ability to manage associated demands. In contrast, we observed 
that individuals who suffer from loneliness are likely to perceive deficits in the ability to manage social and emotional demands. It is therefore possible that loneliness is a useful explanatory factor in self appraisal of social adaptation, which likely helps to explain the effect loneliness exerts on social outcomes such as peer isolation and victimization.

Results regarding socio-demographic variables were noteworthy. Beginning with gender, prior research showed similarity in loneliness in childhood [10], contrasted by equivocal results in high school $[4,15]$. The present study included a developmental period just prior to the transition from junior High to middle High school, and results indicated that young adolescent females reported substantially higher levels of loneliness than their male peers. Furthermore, females experiencing elevated levels of loneliness at baseline suffered the largest reductions in social self-efficacy by one year on. These data show that loneliness places a greater risk on social self efficacy and perhaps related social outcomes for females than males during the onset of adolescence. SES also had an effect on loneliness, though the effect was small. Specifically, students who received free lunch reported modestly higher levels of loneliness than their more affluent peers. However, there was no interaction between SES and loneliness in the prediction of social self-efficacy.

The present study is not without limitations. First, results obtained in one European region may not generalize to adolescents living in other European countries, or other parts of the world more broadly. Second, constructs other than the ones included in the present study have been shown to be associated with loneliness, namely psychological well-being and adjustment. The aforementioned shortcoming provides potentially fruitful lines of inquiry for future research on the short form. Given research on addictive behaviors [30], it would also be important to know the degree to which UCLA loneliness short form scores predict alcohol and substance use during adolescence.

\section{References}

1. Moksnes Unni K, Inger EO Moljord, Geir A Espnes, Don G. Byrne (2010) The association between stress and emotional states in adolescents: The role of gender and self-esteem. Personality and Individual Differences 49: 430-435.

2. Goossens Luc (2006) Affect, emotion, and loneliness in adolescence. In Jackson S, Goossens L (Eds), Handbook of adolescent development. Psychology Press, Hove, UK pp: 51-70.

3. Peplau Letitia A, Daniel Perlman (1982) Perspectives on loneliness. Loneliness: A sourcebook of current theory, research and therapy pp. 1-20.

4. Vanhalst Janne, Luc Goossens, Koen Luyckx, Ron HJ Scholte, Rutger CME Engels (2013) The development of loneliness from mid-to late adolescence: Trajectory classes, personality traits, and psychosocial functioning. Journal of adolescence 36: 1305-1312.

5. Heinrich Liesl M, Eleonora Gullone (2006) The clinical significance of loneliness: A literature review. Clinical psychology review 26: 695-718.

6. West Donald A, Robert Kellner, Maggi Moore-West (1986) The effects of loneliness: a review of the literature. Comprehensive psychiatry 27: 351-363.

7. Hawkley Louise C, John T. Cacioppo (2010) Loneliness matters: a theoretical and empirical review of consequences and mechanisms. Annals of Behavioral Medicine 40: $218-227$.

8. Qualter Pamela, Janne Vanhalst, Rebecca Harris, Eeske Van Roekel, Gerine Lodder, et al. (2015) Loneliness across the life span. Perspectives on Psychological Science 10: $250-264$.
9. Vanhalst, Janne, Koen Luyckx, Luc Goossens (2014) Experiencing loneliness in adolescence: A matter of individual characteristics, negative peer experiences, or both? Social Development 23: 100-118.

10. Jobe-Shields, Lisa Robert Cohen, Gilbert R. Parra (2011) Patterns of change in children's loneliness: Trajectories from third through fifth grades. Merrill-Palmer Quarterly 57: 25-47.

11. Jones Angela C, Katherine C Schinka, Manfred HM van Dulmen, Robert M. Bossarte, Monica H. Swahn (2011) Changes in loneliness during middle childhood predict risk for adolescent suicidality indirectly through mental health problems. Journal of Clinical Child \& Adolescent Psychology 40: 818-824.

12. Cacioppo Stephanie, Angela J Grippo, Sarah London, Luc Goossens, John T. Cacioppo (2015) Loneliness: Clinical import and interventions. Perspectives on Psychological Science 10: 238-249.

13. Qualter Pamela, Stephen Lloyd Brown, KJ Rotenberg, Janne Vanhalst RA Harris, Luc Goossens M Bangee, et al. (2013) Trajectories of loneliness during childhood and adolescence: Predictors and health outcomes. Journal of Adolescence 36: 1283-1293.

14. Qualter Pamela, Stephen L Brown, Penny Munn, Ken J. Rotenberg (2010) Childhood loneliness as a predictor of adolescent depressive symptoms: an 8-year longitudinal study. European Child \& Adolescent Psychiatry 19: 493-501.

15. Benner Aprile D (2011) Latino adolescents' loneliness, academic performance, and the buffering nature of friendships. Journal of Youth and Adolescence 40: 556-567.

16. Wols A, RHJ Scholte, P Qualter (2015) Prospective associations between loneliness and emotional intelligence. Journal of adolescence 39: 40-48.

17. Harris Rebecca A, Pamela Qualter, Sarita J. Robinson (2013) Loneliness trajectories from middle childhood to pre-adolescence: impact on perceived health and sleep disturbance. Journal of Adolescence 36: 1295-1304.

18. Hawkley Louise C, Yuanyuan Gu, Yue-Jia Luo, John T. Cacioppo (2012) The mental representation of social connections: Generalizability extended to Beijing adults. PLoS One 7: e44065.

19. Xinyin Chen, Yunfeng He, Ana Maria De Oliveira, Alida Lo Coco, Carla Zappulla, et al. (2004) Loneliness and social adaptation in Brazilian, Canadian, Chinese and Italian children: a multi-national comparative study.The Journal of Child Psychology and Psychiatry 45: 1373-1384.

20. Van Staden, Werdie CW, Kobus Coetzee (2010) Conceptual relations between loneliness and culture. Current Opinion in Psychiatry 23: 524-529.

21. Bandura Albert (1986) Social foundations of thought andaction. New York: prentice-hall.

22. Bandura Albert (1994) Self-efficacy. In. VS Ramachaudran. Encyclopedia of human behavior 4: 71-81.

23. Bandura Albert (1997) Self-efficacy: The exercise of control. Macmillan.

24. Hughes Mary Elizabeth, Linda J. Waite, Louise C. Hawkley, John T. Cacioppo (2004) A short scale for measuring loneliness in large surveys: Results from two populationbased studies. Research on aging 26: 655-672.

25. Hobbs Graham, Anna Vignoles (2007) Is free school meal status a valid proxy for socio-economic status (in schools research)? Centre for the Economics of Education, London School of Economics and Political Science.

26. Muthén LK, Muthén BO (2016) Mplus User's Guide. ( $7^{\text {th }}$ Edn). (1998-2015). Pape, Hilde, and Thor Norström. Associations between emotional distress and heavy drinking among young people: A longitudinal study. Drug and alcohol review 35: 170-176.

27. Hu Li-tze, Peter M. Bentler (1999) Cutoff criteria for fit indexes in covariance structure analysis: Conventional criteria versus new alternatives. Structural equation modeling: a multidisciplinary journal 6: 1-55.

28. Kelloway E. Kevin (2014) Using Mplus for structural equation modeling: A researcher's guide. Sage Publications.

29. Ferguson Christopher J (2009) An effect size primer: A guide for clinicians and researchers. Professional Psychology: Research and Practice 40: 532

30. Stickley Andrew, Ai Koyanagi, Roman Koposov, Mary Schwab-Stone, Vladislav Ruchkin (2014) Loneliness and health risk behaviours among Russian and US adolescents: a cross-sectional study. BMC public health 14: 366 .

Copyright: (C2018 Andretta JR. This is an open-access article distributed under the terms of the Creative Commons Attribution License, which permits unrestricted use, distribution, and reproduction in any medium, provided the original author and source are credited. 